\title{
Continuous group and electron-count rules in aromaticity
}

\author{
PRADEEP KUMAR* (1) \\ Department of Chemistry, Malaviya National Institute of Technology, Jaipur, Rajasthan 302 017, India \\ E-mail: pradeep.chy@mnit.ac.in
}

MS received 10 September 2017; revised 25 November 2017; accepted 16 December 2017; published online 7 February 2018

\begin{abstract}
In the present article a group theoretical approach has been used to explain different electron-count rules for aromaticity. A general group theoretical method is presented to derive and unite the different electron count rules (Hückel, Baird, Möbius, and Spherical aromaticity). It is shown that continuous groups play important role in understanding of these electron count rules.
\end{abstract}

Keywords. Aromaticity; group theory; continuous groups.

\section{Introduction}

In 1855 August Wilhelm Hofmann used the word 'aromatic' to classify a group of molecules with certain particular properties. ${ }^{1}$ Much before quantum mechanics and even the notion of atom, August Kekule tried to explain those peculiar properties by proposing the resonance structures of benzene, which played a pivotal role in the development of the concept of aromaticity. ${ }^{2}$

Since then, it has been an important concept in chemistry. ${ }^{3-5}$ It was traditionally attributed to the organic compounds. Initially, it was invoked to explain certain non-trivial reactivity and stability of certain compounds. For example, why does benzene undergo electrophilic substitution rather than addition like other alkenes. In modern science, the notion of aromaticity is no longer limited to organic compounds; it has now been already incorporated in other domains like metal clusters, ${ }^{6-8}$ fullerenes, ${ }^{9,10}$ photochemistry ${ }^{11}$ and hydrogen bonded complexes. ${ }^{12}$

The concept of aromaticity was initially confined to the delocalization of electrons in pi $(\pi)$ orbitals, but now, it is known that electrons not only get delocalized in $\pi$ orbitals but also in sigma $(\sigma)$ orbitals, hybrid orbitals and even in delta orbitals. ${ }^{13,14}$ Besides orbitals, the geometry of molecule can also lead to different types of aromaticities. The Möbius aromaticity ${ }^{15-19}$ can be a typical example. In spite of being found routinely in all elementary texts of organic chemistry, aromaticity is still a poorly understood and controversial concept. ${ }^{20}$ One of the reasons might be that aromaticity is not a directly measurable property. ${ }^{21}$ It manifests itself, in

\footnotetext{
*For correspondence
}

terms of changes in structure, reactivity and stability of compounds. Consequently, a number of different indirect measures of aromaticity exist in literature like resonance energy, geometry based index, ${ }^{22}$ information theoretic index, ${ }^{23}$ nuclear independent chemical shift (NICS), ${ }^{24}$ aromatic fluctuation index (FLU), etc. ${ }^{25,26}$ It is generally an accepted fact that all kinds of aromaticities result from delocalization of electrons and all the indexes usually indicate the extent of this delocalization. Since the beginning, besides aromaticity index, electron-count rules were also used to identify the aromatic character of molecules. ${ }^{27}$ The electron count rules usually give only qualitative description of aromaticity, while aromaticity indexes were designed to predict the aromaticity quantitatively. For example according to Hückel rule, ${ }^{3}$ any planar cyclic molecule with $4 N+$ 2 ( $N$ being a positive integer) $\pi$-conjugated electrons is called aromatic. Consequently, benzene and toluene both are aromatic according to Hückel rule, but $4 N+2$ rule does not differentiate between them, whereas values of aromatic indexes, like rig current and resonance energy do differ for benzene and toluene. As soon as aromaticity in inorganic compounds had started to surface, a search for similar electron count rules like Hückel rule also started. For example, when fullerene was discovered and found to have some unusual properties like benzene, researchers tried to explain it on the basis of spherical delocalization of electrons. ${ }^{9,10}$ It was obvious that Hückel rule can not be applied in fullerene or more generally to spherical molecules. Hirsch and co workers formulated the $2(N+1)^{2}$ rules for spherical aromatic compounds. ${ }^{28}$ Similarly, when aromaticity was found in Möbius type molecules, a $4 N$ rules for Möbius type molecules ${ }^{15-17}$ had been formulated. 
Similar to close shell molecules, these electron count rule have also been formulated for open shell systems. Baird ${ }^{29}$ was the first to formulate the $4 N$ rules for planar cyclic open-shell compounds similar to Hückel rule. After the discovery of spherical aromaticity in fullerene like molecules, Poater and Solá ${ }^{30}$ had successfully formulated the $2 N^{2}+2 N+1$ rule for aromaticity in open shell spherical molecules. It warrants a mention here that stating the aromaticity rule does not always mean a complete understanding of the origin of these rules. For example, Hückel proposed his rule based on his LCAOMO calculation which is popularly known as Hückel molecular orbital theory (HMO). Later Dewar ${ }^{31}$ in the mid-1960s used perturbation theory to qualitatively derive and compare the formation energies to explain the Hückel rule. Recently, Miliordos explained the Hückel rule based on a particle on a cylinder model. ${ }^{32}$ It is worth mentioning that Hückel molecular orbital theory was traditionally related to connectivity, therefore topological approach like graph theory gained popularity in explaning Hückel $4 N+2$ rule. In this context, explaining Hückel rule based on a particle on a cylinder model gives a new way of thinking about aromaticity. In another example, although Heilbronner gave the $4 N$ rule for aromaticity in Möbius type molecules ${ }^{15}$ based on simple HMO theory and later H. E. Zimmerman coined the term Möbius aromaticity, ${ }^{16,17}$ a lot of effort was made in the recent past to understand the real origin of this $4 N$ rule. Miliordos solved a particle on Möbius strip problem ${ }^{32,33}$ to unravel the mystery behind $4 N$ rule, whereas McKee et al. ${ }^{34}$ used a more sophisticated HMO approach to understand this rule.

Initially, most of these rules were formed based on explicit $a b$ initio calculations on different molecules. It is important to understand that these electron count rules are very general, as they just use a general kind of symmetry description of molecules. Therefore, instead for using ab initio approaches to explain these rules, symmetry based approaches might be more natural. Another importance of using the symmetry based approach is, it can give a connection between different electron count rules. For example, Rubin and Ordóñez ${ }^{35}$ computed the eigenvalues and degeneracy of general $n$-dimensional spherical harmonics. One can clearly see there, $n=2$ case corresponds to Hückel rule, whereas $n=3$ case corresponds to Hirsch rule. In another case Poater et al., ${ }^{36}$ used electronic confined space analogy (ECSA) to show that $4 N+2$ Wade-Mingos rule ${ }^{37,38}$ for three dimensional closo boranes is equivalent to the $4 N+2$ Hückel rule for polycyclic aromatic hydrocorbon.

Symmetry based approaches are nowadays gaining popularity in explaining these electron count rules. ${ }^{39-41}$ B. A. Shainyan ${ }^{41}$ used a physical symmetry, which he called symmetry of space of states to shed light on the origin of electron count rules. Recently, it was shown that tubular aromaticity found in tubular boron clusters can be explained using particle in hollow cylinder model. ${ }^{42,43}$ Same group used particle on a disk model, to explain aromaticity in planner boron cluster and coined the term disk aromaticity. ${ }^{44-46}$ It is important to note that explaining aromaticity by solving classical text book problems like particle on sphere, ${ }^{28}$ particle on disk, ${ }^{45,46}$ particle on hollow cylinder ${ }^{42,43}$ or particle on Möbius strip $^{32,33}$ are essentially symmetry based approaches. In all these problems, authors were trying to utilize, the underlying symmetry involved in electron delocalization space. Therefore, in explaining aromaticity rules, it appears natural to utilize the symmetry, directly, using group theory rather than solving differential equations.

In the present paper, the author explains different electron count rules, using the symmetry arguments based on group theory. The approach used here, is based on continuous groups, rather than point groups. Hopefully, the present work will complement other works that used symmetry based argument directly or indirectly, to explain aromaticity and will strengthen the symmetry based arguments, in exploring aromaticity.

\section{Theory}

Our chemical intuition says, that, aromaticity is actually the concept of extensive delocalization of electrons. The symmetry group of molecule, will depend on the structure of molecule. But, when electrons get truly delocalized, they will be in continuous symmetry. Consequently, when a molecule is aromatic, then the relevant electrons live in a different symmetry space. This delocalization space must be some continuous symmetry space. Therefore, continuous groups, not point groups should play important role in understanding of electron count-rules. It makes the study of aromaticity simple. Instead of solving full eigenvalue problems of actual Hamiltonians, one should solve text book kind of problems of continuous symmetry. For example, particle on a cylinder, particle on a sphere, etc. As mentioned in the introduction, similar approaches have been used to explain Hückel, Möbius, tubular, spherical and disk aromaticity. It is known that symmetry plays an important role in solving such kind of differential equations. In the context of electron count rules, this symmetry aspect of differential equations, become very important, as we are not looking for exact eigenvalues or eigenfunctions. Rather, we are in search of a general distribution of eigenfunctions. Lot of qualitative features about such kind of problems, can be understood, through group theory, without explicitly solving the differential equation. This can be illustrated by typical text book 
solution of hydrogen atom. Hydrogen atom has a spherically symmetric potential. The angular part of the wave function turns out to be spherical harmonics. It is no coincidence that the spherical harmonics form the irreducible representation of the rotation group (particularly that of special orthogonal group, of order three, normally known as $S O$ (3) group).

In the present article, symmetry based approach is used, instead of solving the differential equations. For the present analysis, there are two important aspects in the explanation of electron-count rules: first, to recognize the symmetry of the space in which electrons get delocalized; second, the symmetry of the orbitals involved in the delocalization process. Once, these two symmetries are recognized, we have to look for the irreducible representations of that group that accommodates these two symmetries. Within that group, the stable configuration corresponds to aromaticity whereas unstable configuration corresponds to antiaromaticity. This general methodology is illustrated in the next section by applying it to various classes of aromaticity.

\section{Results and Discussion}

\subsection{Hückel and Baird aromaticity}

The molecules belonging to this class, have cylindrical symmetry for delocalized electrons. We can represent the cylinder on a two dimensional $\mathrm{R}^{2}$ plane (Figure 1 ) in the rotational angle space $(\phi)$.

In this plane, the cylindrical symmetry corresponds to the translational symmetry in $\phi$. The relevant group for cylindrical symmetry is special orthogonal group of order two i.e., $\mathrm{SO}(2)$, whose irreducible representations can be represented by $\exp (-i m \phi)$ where $m=$ $0, \pm 1, \pm 2$ etc. The values of $m$ are obtained from the boundary condition, which requires that, after a $2 \phi$ translation, a vector should be back onto the original vector. Now, if the orbitals involved in delocalization are $\pi$ orbitals, we can use an inversion symmetry in conjunction with $S O(2)$ symmetry. Consequently the resultant symmetry group will be a direct product of $S O(2)$ and

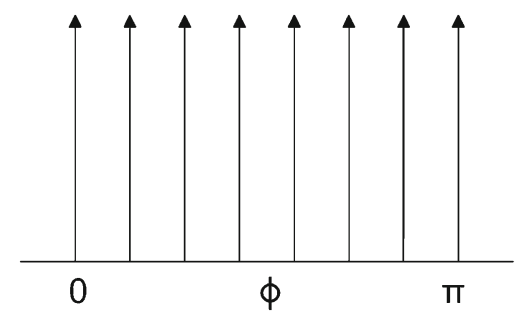

Figure 1. The representation of cylinder in 2-d Space. The vectors are used to indicate the different points on cylinder's cross section. $\sigma_{h}$ i.e., $S O(2) \otimes \sigma_{h}$, which will be $D_{\infty h}$ (after including inversion) group. The character table for $\mathrm{D}_{\infty h}$ point group is shown in Table 1.

This point group has two sets of, one dimensional as well as, two dimensional representations. One set with positive character for inversion $(\mathrm{g})$ and other one with negative character $(\mathrm{u})$. The $\pi$ orbitals will belong to ungerade (u) set of one as well as two dimensional representations. The molecular orbital diagram, under this symmetry, is shown in Figure 2.

Now, if we are dealing with a closed shell molecule, we need a closed shell configuration for these orbitals. Using Pauli exclusion principle to fill up these orbitals, the closed shell configuration will corresponds to $4 N+2$ electrons for $2 N+1$ orbitals. Therefore, $4 N+2$ electrons are very natural for these closed shell molecules, in which electrons get delocalized within cylindrical space. It is interesting to note that, in molecules where more than one layer of atoms exist (Instead of molecules having a ring topology, they have a disk or tubular structure), more than one such set of orbitals may exist. Quantum mechanically it means, additional quantum numbers exist, besides the angular one. Consequently, the closed configuration can consist of $4 N+2$ (Figure $3 \mathrm{a}$ ), or $4 N$ (Figure $3 \mathrm{~b}$ ), depending upon the exact energy ordering of molecular orbitals.

This discussion, can easily be extended to the case, where electrons get delocalized in $\sigma$ orbitals ( $\sigma$ aromaticity). Here again, one can use the $D_{\infty h}$ point group, to derive the aromaticity rule. The rule for $\sigma$ aromaticity will be the same, except the fact that $\sigma$ orbital will correspond to the gerade $(\mathrm{g})$ set of one as well as two dimensional representations. Consequently, electron count rule will be the same regardless of whether electron gets delocalized in $\sigma$ or in $\pi$ orbitals. The $\sigma$ aromaticity and $4 N$ rule are not very apparent in organic molecules, as organic molecules usually have a ring topology and $\pi$ delocalization. But in inorganic molecules, one can see it. For example, depending upon the number of boron atoms and overall charge, boron clusters can exist both in disk form and in tubular form. ${ }^{47}$ There, depending upon the exact ordering of molecular orbitals the stable boron cluster may consist of either $4 N+2$ or $4 N$ electrons. ${ }^{42-46}$

In case of open shell molecules, one needs to fill these molecular orbitals, in such a way that the resulting configuration need to be consistent with spin state of molecule. If the molecule is in triplet state, then total number of electrons will be $4 N+2+2$, where extra two electrons are unpaired electrons. So essentially, $4 N$ electrons will be optimal for both triplet and singlet states to exhibit aromaticity, famously known as Baird's rule. 
Table 1. In the following table the symbols and numbers to the left and above the double lines refer to the $D_{\infty h}$ point group. Below and to the right of the broken lines are species and characters of corresponding double group $\left(D_{\infty h}^{*}\right)$. The $\mathrm{E}(2 \pi)$ represents the $2 \pi$ rotation operator while $\mathrm{E}(4 \pi)$ is the $2 \pi$ rotation operator.

\begin{tabular}{ccccccccccc}
\hline & $\mathrm{E}(2 \pi)$ & $2 \mathrm{C}_{\infty}$ & $\ldots$ & $\infty \sigma_{v}$ & $\mathrm{i}$ & $2 \mathrm{~S}_{\infty}$ & $\ldots$ & $\infty \mathrm{C}_{2}^{\prime}$ & $\mathrm{E}(4 \pi)$ & $\ldots$ \\
\hline$\Sigma_{g}^{+}$ & 1 & 1 & $\ldots$ & 1 & 1 & 1 & $\ldots$ & 1 & 1 & $\ldots$ \\
$\Sigma_{g}^{-}$ & 1 & 1 & $\ldots$ & -1 & 1 & 1 & $\ldots$ & -1 & 1 & $\ldots$ \\
$\Pi_{g}$ & 2 & $2 \cos (\phi)$ & $\ldots$ & 0 & 2 & $-2 \cos (\phi)$ & $\ldots$ & 0 & 2 & $\ldots$ \\
$\Delta_{g}$ & 2 & $2 \cos (2 \phi)$ & $\ldots$ & 0 & 2 & $2 \cos (2 \phi)$ & $\ldots$ & 0 & 2 & $\ldots$ \\
$\ldots$ & $\ldots$ & $\ldots$ & $\ldots$ & $\ldots$ & $\ldots$ & $\ldots$ & $\ldots$ & $\ldots$ & & $\ldots$ \\
$\Sigma_{u}^{+}$ & 1 & 1 & $\ldots$ & 1 & -1 & -1 & $\ldots$ & -1 & 1 & $\ldots$ \\
$\Sigma_{u}^{-}$ & 1 & 1 & $\ldots$ & -1 & -1 & -1 & $\ldots$ & 1 & 1 & $\ldots$ \\
$\Pi_{u}$ & 2 & $2 \cos (\phi)$ & $\ldots$ & 0 & -2 & $2 \cos (\phi)$ & $\ldots$ & 0 & 2 & $\ldots$ \\
$\Delta_{u}$ & 2 & $2 \cos (2 \phi)$ & $\ldots$ & 0 & -2 & $-2 \cos (2 \phi)$ & $\ldots$ & 0 & 2 & $\ldots$ \\
$\ldots$ & $\ldots$ & $\ldots$ & $\ldots$ & $\ldots$ & $\ldots$ & $\ldots$ & $\ldots$ & $\ldots$ & & $\ldots$ \\
$E_{\frac{1}{2} g} g$ & -2 & $2 \cos (2 \phi / 2)$ & $\ldots$ & 0 & 2 & $\ldots$ & $\ldots$ & $\ldots$ & 2 & $\ldots$ \\
$E_{\frac{3}{2} g} g$ & -2 & $2 \cos (3 \phi / 2)$ & $\ldots$ & 0 & 2 & $\ldots$ & $\ldots$ & $\ldots$ & 2 & $\ldots$ \\
$E_{\frac{5}{2} g} g$ & -2 & $2 \cos (5 \phi / 2)$ & $\ldots$ & 0 & 2 & $\ldots$ & $\ldots$ & $\ldots$ & 2 & $\ldots$ \\
$\ldots$ & $\ldots$ & $\ldots$ & $\ldots$ & $\ldots$ & $\ldots$ & $\ldots$ & $\ldots$ & $\ldots$ & & $\ldots$ \\
\hline
\end{tabular}
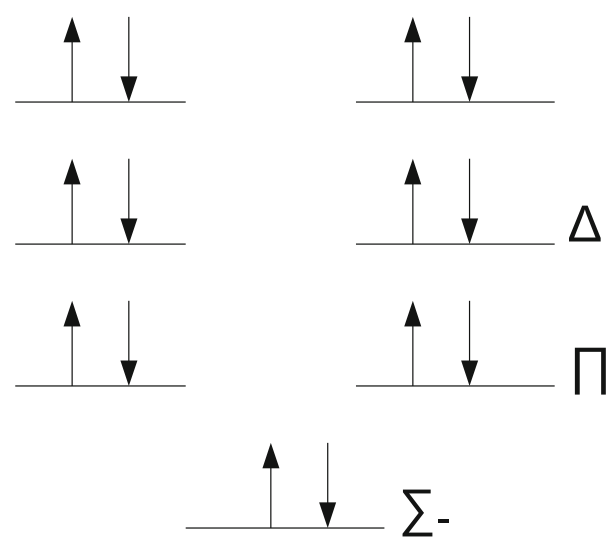

\section{$\mathrm{D}_{\infty \mathrm{h}} \quad 4 \mathrm{~N}+2$}

Figure 2. Molecular orbital diagram for electrons on cylinder.

\subsection{Möbius aromaticity}

Molecules belongs to this category have a Möbius topology. Although, theoretical prediction about Möbius aromaticity came much earlier, experimental observation of such molecules are still an active area of research. ${ }^{18,19,48}$ Initially, the search for Möbius aromaticity were limited to annulenes, but recently, expanded porphyrins is gaining popularity ${ }^{19,49}$ as potential candidates for Möbius aromaticity. The parametric equation for Möbius strip is as follows. ${ }^{33}$

$x=[R+s \cos ( \pm \phi)] \cos (\phi)$

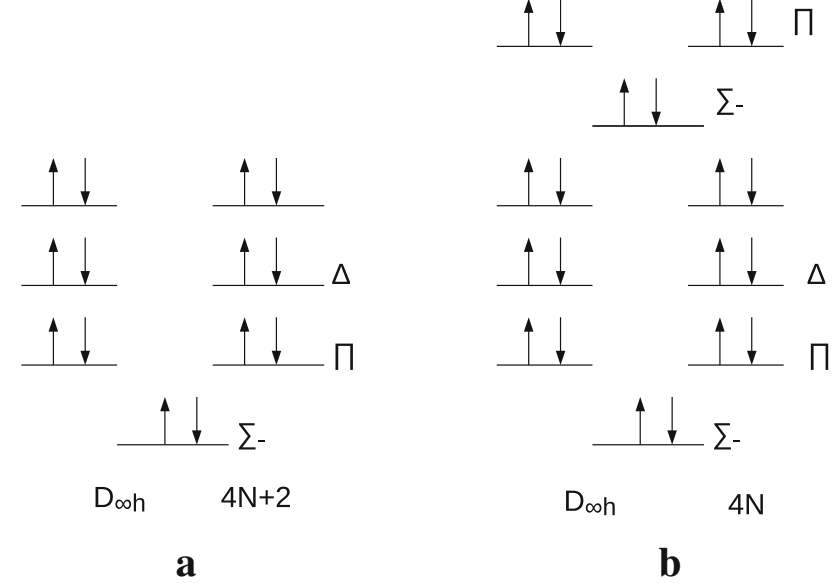

Figure 3. Molecular orbital diagram for electrons on cylinder. (a) represents an arrangement where $4 N+2$ rules applicable whereas (b) represents a configuration which complies $4 N$ rule.

$y=[R+s \cos ( \pm \phi)] \sin (\phi)$

$z=s\left[\sin \left(\frac{\phi}{2}\right)\right]$

If we wish to represent it on a $\mathrm{R}^{2}$ plane, it will look like Figure 4. It is evident from Figure 4, that the vectors not only translate, but also twist.

The translation in $\phi$ direction and translation in $z$ direction (which give rise to the twisting) can be separated. Therefore, the representations of Möbius space symmetry have two parts; one comes from invariance of translation in $\phi$ direction, and, the other one comes 


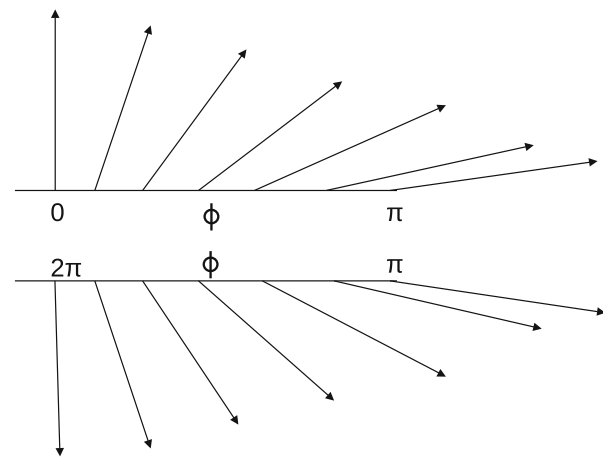

Figure 4. The Möbius strip in 2-d Space. The vectors are used to indicate the different points on Möbius strip.

from invariance of translation in $z$ direction. Mathematically, it will be of the form $\mathrm{e}^{ \pm i m \phi} \mathrm{e}^{ \pm i c m z}$. The particle on the Möbius strip has been solved analytically and the constant ' $c$ ' was found to be $2 / \mathrm{R} .{ }^{33}$ The cylindrical symmetry can be applied to Möbius strip, with one major modification to the Hückel case; in case of Möbius strip the boundary condition involves a rotation of $4 \pi$, not $2 \pi$. Due to this boundary condition, $m$ can take values $0, \pm \frac{1}{2}, \pm 1, \pm \frac{3}{2}, \pm 2$, etc. The half integer values of $m$ make the Möbius strip interesting. In the case of Möbius strip, one should use the double group of $D_{\infty h}$, which means, the group relevant to Möbius aromaticity will be $D_{\infty h} \otimes$ spin-group. The double group for $D_{\infty h}$ is given in Table $1{ }^{50}$ For double group, the identity element is $4 \pi$ rotation, not $2 \pi$ rotation. If electron delocalization takes place through $\pi$ orbitals, we have to search for, that irreducible representation of this group in which $\pi$ orbital belongs. On the Möbius strip, a $2 \pi$ rotation will change the phase of $\pi$ orbital, that means the character corresponding to the $2 \pi$ rotation will be negative. If we see the characters of $\mathrm{D}_{\infty h}$ and the corresponding double group, it is evident that, the representations, which have negative character value, corresponds to half-integer values of $m$, not full integer values. It is clear from the orbital digram given in Figure 5, aromaticity rule for Möbius strip, will be $4 N$ ( $2 N$ orbital), as this give rise to a closed configuration.

It is important to note that, this rule is for $\pi$ aromaticity.

The $\sigma$ orbital do not change phase on $2 \pi$ rotation, hence it belongs to full as well as half-integer value of $m$. Consequently, the $\sigma$-aromaticity rule for Möbius strip will be $4 N+2$ as it was for Hückel case.

\subsection{Spherical aromaticity}

For spherical molecules like fullerene, electron delocalization space is a sphere, which belongs to $S O(3)$

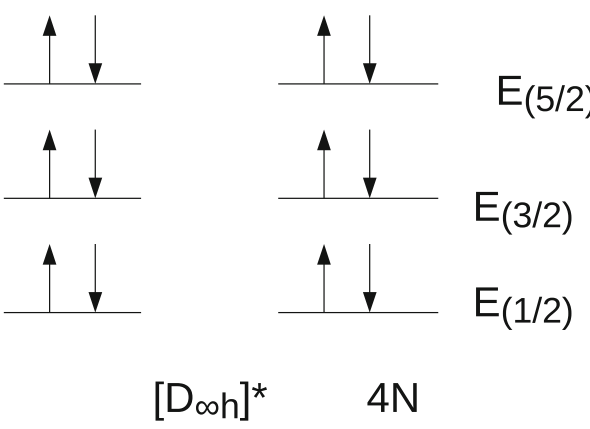

Figure 5. Molecular orbital diagram for electrons on Möbius strip.

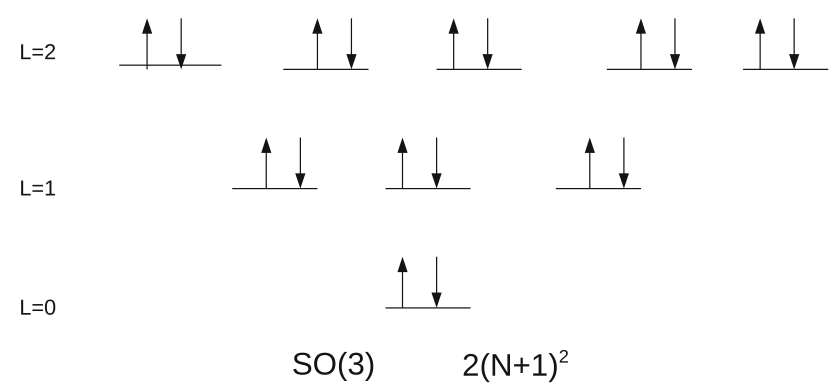

Figure 6. Molecular orbital diagram for electrons on sphere.

symmetry group. If we include inversion symmetry, the relevant group become $\mathrm{SO}(3) \otimes \mathrm{C}_{i}$, which is the $\mathrm{O}(3)$ group. The spherical harmonics can be used to represent the irreducible representations of this group. It is evident from Figure 6 that, in this case, the orbitals will be counted as $1,3,5,7--$, which will be total $(N+1)^{2}$ orbitals where $N$ is an integer.

If we fill electrons, using Pauli principle, the closed shell will be formed with $2(N+1)^{2}$ electrons. Therefore, the electron-count rule for spherical molecules will be $2(N+1)^{2}$. Let us analyze the open shell spherical molecule. In this case, when HOMO is half filled, then it corresponds to a stable configuration i.e., aromatic. If we count, this number will be $1,5,13,25$ - which mean $2 N^{2}+2 N+1$ for an electron spin of $S=N+\frac{1}{2}$.

Three dimensional aromaticity in literature can be divided into the two groups one as "Spherical aromaticity" and other one as "Spatial aromaticity". 7,8,41,51,52 The word spherical aromaticity is reserved for fullerene like molecules which obeys strictly $2(N+1)^{2}$ and $2 N^{2}+2 N+1$ rule for closed shell and open shell systems respectively, whereas word spatial aromaticity is applicable to various cage-like compounds. As mentioned earlier, the tubular aromaticity, which is a spatial aromaticity, obeys the generalized Hückel rule. In this context, the $S O(3)$ group is useful only for spherical aromaticity not for spatial aromaticity. Most of the times 
one needs to map the spatial aromaticity to some continuous symmetry. For example, as mentioned in the introduction, Poater et al., ${ }^{36}$ had successfully mapped the three dimensional aromaticity in closo borane to Hückel like cylindrical symmetry space.

\section{Conclusion}

Based on the group theory, the present analysis concludes that the origin of all electron-count rules are the same. It assumes that, electrons are moving freely in the molecules. Therefore, continuous groups play an important role in deciding the electron-count rules. It was also observed that these electron count rules also depend on the type of orbitals. For example, in case of Möbius aromaticity electron count rules are opposite for $\sigma$ and $\pi$ orbitals. Group theoretical methods give a nice way to incorporate the symmetry of orbitals into the electron delocalization symmetry space. Here, we have successfully classified different types of aromaticities in terms of irreducible representations of continuous groups, except spatial aromaticity.

Finally, It is also worth mentioning that Group theory is a well-developed mathematical theory. Therefore, we believe that besides unifying different electron-count rules, it will be useful to shed more light on different questions related to aromaticity. For example, to derive electron-count rules, the present analysis assumes a complete delocalization of electron, but we know that, aromaticity per se does not required a complete delocalization. There are also incidence that a perturbation can increase or decrease the aromaticity character of system. In such cases, whether Group theory can be helpful or not, is a matter of further investigation.

\section{Acknowledgements}

Author acknowledges the financial support from SERBDST, Govt. of India through the Project No. ECR/2016/ 000279 .

\section{References}

1. Hofmann A W 1856 On insolinic acid Proc. R. Soc. Lond. 81

2. Kekulé A 1865 Sur la constitution des substances aromatiques Bull. Soc. Chim. Fr. (Paris) 398

3. Clayden J, Greeves N, Warren S and Wothers P 2001 Organic Chemistry (New York: Oxford University Press)

4. Schleyer P von R 2011 Introduction: Aromaticity Chem. Rev. 1011115

5. Garrat P J 1986 Aromaticity (New York: Wiley)

6. Boldyrev A I and Wang L S 2005 All-metal aromaticity and antiaromaticity Chem. Rev. 1053716
7. King R B 2001 Three-dimensional aromaticity in polyhedral boranes and related molecules Chem. Rev. 101 1119

8. Chen Z and King R B 2005 Spherical aromaticity: recent work on fullerenes, polyhedral boranes, and related structures Chem. Rev. 1053613

9. Bühl M and Hirsch A 2001 Spherical aromaticity of fullerenes Chem. Rev. 1011153

10. Garcia-Borràs $\mathrm{M}$, Osuna $\mathrm{S}$, Luis $\mathrm{J} \mathrm{M}$, Swart $\mathrm{M}$ and Solà M 2014 The role of aromaticity in determining the molecular structure and reactivity of (endohedral metallo) fullerenes Chem. Soc. Rev. 435089

11. Ottosson H 2012 Organic photochemistry: Exciting excited-state aromaticity Nat. Chem. 4969

12. Datta A and Pati S K 2006 Stability of cyclic $\left(\mathrm{H}_{2} \mathrm{O}\right)_{n}$ clusters within molecular solids: Role of aromaticity Int. J. Quantum Chem. 1061697

13. Zubarev D Y, Averkiev B B, Zhai H J, Wang L S and Boldyrev A I 2008 Aromaticity and antiaromaticity in transition-metal systems Phys. Chem. Chem. Phys. 10 257

14. Yu X, Oganov A R, Popov I A and Boldyrev A I 2016 d-AO spherical aromaticity in $\mathrm{Ce}_{6} \mathrm{O}_{8} J$. Comput. Chem. 37103

15. Heilbronner E 1964 Hückel molecular orbitals of Möbius-type conformations of annulenes Tetrahedron Lett. 51923

16. Zimmerman H E 1966 On molecular orbital correlation diagrams, the occurrence of Möbius systems in cyclization reactions, and factors controlling ground-and excited-state reactions J. Am. Chem. Soc. 881564

17. Zimmerman H E 1971 Möbius-Hückel concept in organic chemistry. Application of organic molecules and reactions Acc. Chem. Res. 4272

18. Rzepa H S 2005 Möbius aromaticity and delocalization Chem. Rev. 1053697

19. Yoon Z S, Osuka A and Kim D 2009 Möbius aromaticity and antiaromaticity in expanded porphyrins Nat. Chem. 1113

20. Cyranski M K, Krygowski T M, Katritzky A R and Schleyer P v R 2002 To what extent can aromaticity be defined uniquely? J. Org. Chem. 671333

21. Katritzky A R, Karelson M, Sild S, Krygowski T M and Jug K 1998 Aromaticity as a quantitative concept. 7. Aromaticity reaffirmed as a multidimensional characteristic J. Org. Chem. 635228

22. Krygowski $\mathrm{T}$ M, Szatylowicz H, Stasyuk O A, Dominikowska J and Palusiak M 2014 Aromaticity from the viewpoint of molecular geometry: Application to planar systems Chem. Rev. 1146383

23. Noorizadeh $S$ and Shakerzadeh E. 2010 Shannon entropy as a new measure of aromaticity, Shannon aromaticity, Shannon aromaticity Phys. Chem. Chem. Phys. 124742

24. Chen Z, Wannere C S, Corminboeuf C, Puchta R and Schleyer P v R 2005 Nucleus-independent chemical shifts (NICS) as an aromaticity criterion Chem. Rev. 105 3842

25. Matito E, Duran M and Sola M 2005 The aromatic fluctuation index (FLU): A new aromaticity index based on electron delocalization J. Chem. Phys. 122014109

26. Katritzky A R, Jug K and Oniciu D C 2001 Quantitative measures of aromaticity for mono-, bi-, and tricyclic 
penta-and hexaatomic heteroaromatic ring systems and their interrelationships Chem. Rev. 1011421

27. Feixas F, Matito E, Poater J and Solà M 2016 In Applications of Topological Methods in Molecular Chemistry R Chauvin, C Lepetit, B Silvi and E Alikhani (Eds.) (Switzerland: Springer International Publishing) p. 321

28. Hirsch A, Chen Z and Jiao H 2000 Spherical aromaticity in Ih symmetrical fullerenes: The $2(N+1)^{2}$ rule Angew. Chem. Int. Edit. 393915

29. Baird N C 1972 Quantum organic photochemistry. II. Resonance and aromaticity in the lowest 3. pi.. pi.* state of cyclic hydrocarbons J. Am. Chem. Soc. 944941

30. Poater J and Solà M 2011 Open-shell spherical aromaticity: The $2 N^{2}+2 N+1$ (with $S=N+1 / 2$ ) rule Chem. Commun. 4711647

31. Dewar M J S 1966 A molecular orbital theory of organic chemistry VIII: Aromaticity and electrocyclic reactions Tetrahedron Suppl 22 (Suppl. 8) 75

32. Miliordos E 2010 Hückel versus Möbius aromaticity: The particle in a cylinder versus a Möbius strip Phys. Rev. A 82062118

33. Miliordos E 2011 Particle in a Möbius wire and halfinteger orbital angular momentum Phys. Rev. A 83 062107

34. McKee W C, Wu I.Judy, Rzepa H S. and Schleyer P v R 2013 A Hückel theory perspective on Möbius aromaticity Organ. Lett. 153432

35. Rubin M A and Ordónez C R 1984 Eigenvalues and degeneracies for n-dimensional tensor spherical harmonics J. Math. Phys. 252888

36. Poater J, Solà M, Viñas C and Teixidor F $2014 \pi$ Aromaticity and Three-Dimensional Aromaticity: Two sides of the Same Coin? Angew. Chem. Int. Edit. 5312191

37. Wade K 1971 The structural significance of the number of skeletal bonding electron-pairs in carboranes, the higher boranes and borane anions, and various transitionmetal carbonyl cluster compounds J. Chem. Soc. D 792

38. Mingos D M P 1972 A general theory for cluster and ring compounds of the main group and transition elements Nat. Phys. Sci. 23699

39. Fowler P W and Rzepa H S 2006 Aromaticity rules for cycles with arbitrary numbers of half-twists Phys. Chem. Chem. Phys. 81775
40. Goldstein M and Hoffmann R 1971 Symmetry, topology, and aromaticity J. Am. Chem. Soc. 936193

41. Shainyan B A 2011 Electron-counting rules, threedimensional aromaticity, and the boundaries of the Periodic Table J. Phys. Org. Chem. 24619

42. Pham H T, Duong L V and Nguyen M T 2014 Electronic structure and chemical bonding in the double ring tubular boron clusters J. Phys. Chem. C 11824181

43. Van Duong L, Pham H T, Tam N M and Nguyen M T 2014 A particle on a hollow cylinder: the triple ring tubular cluster $\mathrm{B}_{27}{ }^{+}$Phys. Chem. Chem. Phys. 16 19470

44. Tai T B, Ceulemans A and Nguyen M T 2012 Disk aromaticity of the planar and fluxional anionic boron clusters $B_{20}{ }^{-/ 2}-$ Chem. Eur. J. 184510

45. Tai T B, Havenith R W, Teunissen J L, Dok A R, Hallaert S D, Nguyen M T and Ceulemans A 2013 Particle on a boron disk: Ring currents and disk aromaticity in $\mathrm{B}_{20}{ }^{2-}$ Inorg. Chem. 5210595

46. Tai T B, Van Duong L, Pham H T, Mai D T T and Nguyen M T 2014 A disk-aromatic bowl cluster B Tai T B, Van Duong L, Pham H T, Mai D T T and Nguyen M T 2014 A disk-aromatic bowl cluster $\mathrm{B}_{3} 0$ : toward formation of boron buckyballs Chem. Commun. 501558

47. Alexandrova A N, Boldyrev A I, Zhai H J and Wang L-S 2006 All-boron aromatic clusters as potential new inorganic ligands and building blocks in chemistry Coord. Chem. Rev. 2502811

48. Herges R 2006 Topology in chemistry: Designing Möbius molecules Chem. Rev. 1064820

49. Tanaka Y, Saito S, Mori S, Aratani N, Shinokubo H, Shibata N, Higuchi Y, Yoon Z S, Kim K S and Noh S B 2008 Metalation of expanded porphyrins: A chemical trigger used to produce molecular twisting and Möbius aromaticity Angew. Chem. 120693

50. Herzberg G 1966 Electronic Spectra of Polyatomic Molecules (New York: Van Nostrand)

51. Shainyan B. 2006 Rules for counting electrons and three-dimensional aromaticity Russ. J. Organ. Chem. 42 304

52. El Bakouri O, Duran M, Poater J, Feixas F and Solà M 2016 Octahedral aromaticity in ${ }^{2 S+1} A_{1 g} X_{6}{ }^{q}$ clusters $(\mathrm{X}=\mathrm{Li}-\mathrm{C}$ and $\mathrm{Be}-\mathrm{Si}, \mathrm{S}=0-3$, and $\mathrm{q}=-2$ to +4$)$ Phys. Chem. Chem. Phys. 1811700 